\title{
Cuestiones jurídicas controvertidas sobre las técnicas del «Tactical Combat Casualty Care» en la actual legislación española
}

\author{
González-Reyes J. ${ }^{1}$
}

Sanid. mil. 2021; 77 (1): 35-39, ISSN: 1887-8571

\begin{abstract}
RESUMEN
El nuevo concepto TCCC («tactical combat casualty care») ha revolucionado el entorno táctico prehospitalario mejorando de manera exponencial las posibilidades de supervivencia del herido en combate. Tal entendimiento en la atención del trauma bélico, ha supuesto la definitiva aceptación del uso prioritario y preferente de los torniquetes de extremidades y tronconodales, la aplicación de productos hemostáticos capaces de coercer hemorragias masivas, y diferentes recomendaciones y mejoras en la atención sanitaria prehospitalaria actualizadas con la mejor evidencia científica disponible y adaptadas al medio, la misión y las circunstancias en las que se prestan los cuidados en ambiente táctico.

La adecuación de los militares formados en dicho entorno TCCC al ordenamiento jurídico no está exento de cierta complejidad respecto de algunas cuestiones como la legitimación y técnicas de actuación que pueden realizar por tratarse de ámbitos, tradicionalmente, reservados al personal facultativo, especialmente, en lo referente a técnicas invasivas como pueden ser la canalización de accesos periféricos (IV/IO), punción cricotiroidea o administración directa de medicación.
\end{abstract}

PALABRAS CLAVE: TCCC, intrusismo sanitario, eximente, estado de necesidad exculpante.

\section{Disputed legal issues about the techniques of the «Tactical Combat Casualty Care» in current spanish legislation}

\section{SUMMARY}

The new TCCC concept («Tactical Combat Casualty Care») has revolutionized the pre-hospital tactical environment, exponentially improving the survival chances of the casualty in combat. Such understanding in the care of war trauma has led to the definitive acceptance of the priority and preferential use of tourniquets for the extremities and trunks, the application of hemostatic products capable of coercing massive hemorrhages, and different recommendations and improvements in pre-hospital health care updated with the best scientific evidence available and adapted to the environment, mission and circumstances in which care is provided in the tactical environment.

The adaptation of the military trained in said TCCC environment to the legal system is not exempt from certain complexity with respect to some issues such as legitimation and performance techniques that they can carry out because they are areas, traditionally, reserved for medical personnel, especially in relation to to invasive techniques such as peripheral access cannulation (IV / IO), cricothyroid puncture or direct administration of medication.

KEY WORDS: TCCC, sanitary intrusion, exempt, state of need justification

\section{INTRODUCCIÓN}

El nuevo concepto TCCC («tactical combat casualty care») ha revolucionado el entorno táctico prehospitalario mejorando de manera exponencial las posibilidades de supervivencia del herido en combate. Tal entendimiento en la atención del trauma bélico, ha supuesto la definitiva aceptación del uso prioritario y preferente de los torniquetes de extremidades y tronconodales, la aplicación de productos hemostáticos capaces de coercer hemorragias masivas, y diferentes recomendaciones y mejoras en la atención sanitaria prehospitalaria actualizadas con la mejor

\footnotetext{
${ }^{1+}$ Comandante Auditor Doctor en Derecho. Sección Jurídica n. ${ }^{\circ} 11$.

Dirección para correspondencia: José González Reyes. ASEJUSUIGE. Avda. 25 de Julio n. ${ }^{\circ}$ 1. 38003-Santa Cruz de Tenerife.

Recibido: 19 de enero de 2021

Aceptado: 17 de febrero de 2021

doi: $10.4321 /$ S1887-85712021000100006
}

evidencia científica disponible y adaptadas al medio, la misión y las circunstancias en las que se prestan los cuidados en ambiente táctico $^{(1)}$.

La adecuación de los militares formados en dicho entorno TCCC al ordenamiento jurídico no está exento de cierta complejidad respecto de algunas cuestiones como la legitimación y técnicas de actuación que pueden realizar por tratarse de ámbitos, tradicionalmente, reservados al personal facultativo, especialmente, en lo referente a técnicas invasivas como pueden ser la

\footnotetext{
(1) Diferentes estudios revelan que, aproximadamente, el $80 \%$ de las muertes en las operaciones militares ocurren en los 30 minutos a continuación de sufrir las heridas, cuando a menudo el único cuidado del que disponen es el que ellos mismos o un compañero les puede proporcionar. El proceso hasta llegar a la muerte, tras recibir la herida, es el resultado de la pérdida masiva de sangre, en un breve espacio de tiempo, donde el cuerpo sufre un colapso orgánico y deja de funcionar. La terminología médica se refiere a este periodo de tiempo tan urgente como «the golden hour». Cfr., BELLAMY RF. The causes of death in Conventional Land Warfare: Military Medicine 149. February 1984.
} 
canalización de accesos periféricos (IV/IO), punción cricotiroidea o administración directa de medicación.

\section{LA EVIDENCIA CIENTÍFICA}

Un porcentaje significativo de las muertes producidas en las operaciones militares son potencialmente evitables cuanto más inmediata sea la atención sanitaria, como ha demostrado la evidencia científica ${ }^{(2)}$.

Al igual que ocurre en el resto de procesos relacionados con la enfermedad traumática, los de origen bélico comparten el énfasis en realizar una estabilización tempranísima de la baja que permita su supervivencia (hemorragia comprimible, neumotórax a tensión, compromiso en vía aérea y ventilación).

Aunque el trauma en combate es fundamentalmente quirúrgico, en los escenarios donde se desarrollan actualmente estas operaciones se presentan habitualmente situaciones de aislamiento (personal recovery), con bajas múltiples y dispersas sobre el terreno, frecuentemente en ambiente hostil que no pueden ser atendidas de inmediato por personal médico o enfermero, esto es, el denominado personal facultativo y que goza de su propio régimen jurídico estatutario y competencial.

En este contexto, para la salvaguarda de la vida, se impone la necesidad de contar con otro personal militar que, aunque no facultativo, esté capacitado para actuar en estas situaciones, realizando las maniobras pertinentes que estabilicen y permitan la atención posterior por parte de los facultativos, cuya función no sustituyen, y su evacuación a las formaciones sanitarias de tratamiento correspondientes.

\section{MARCO NORMATIVO INTERNACIONAL Y HABILITACIÓN LEGAL}

En las alianzas de las que España forma parte, el apoyo sanitario en las Fuerzas Armadas (FAS) concentra al personal facultativo en formaciones sanitarias de tratamiento ${ }^{(3)}$. En este modelo, las medidas de atención sanitaria inmediata resultan decisivas para la supervivencia del herido y son aplicadas por personal militar no facultativo, aunque adecuadamente instruido y equipado, de acuerdo con protocolos establecidos por las Organizaciones Internacionales a las que España pertenece y ha ratificado en acuerdos de normalización articulando su doctrina sanitaria a partir del MC 326/2 «Nato medical support principles and policies», de la AJP-4.10 (A) «Allied joint medical support doctrine», así como la Directiva de la OTAN «Allied command operations directive for medical support to operations» (AD 83-1) de marzo de 2009.

\footnotetext{
(2) Entre la doctrina que cita la misma puede verse, ampliamente, a BUKOWSKI, CW. The Platinum 10: 2nd BCT, 101st Airborne improves medical training to help save lives. Infantry Magazine, July-August 2006; TARPEY MJ. Tacticcal Combat Casualty Care in Operation Iraqui Freedom. Army Medical Department Journal. June 2005; DE LORENZO RA. 91W: Force XXI Combat Medic. Army Medical Department Journal. December 1999; BUTLER, FKJr. Tactical Combat Casualty Care in Special Operations. Military Medicine 161. 1996.

(3) Vid., ampliamente, Orientaciones de la Sanidad en Operaciones (OR7-603), PMET 3 de mayo de 2004
}

Hay que dotar, entonces, a estos «primeros intervinientes», de una habilitación legal que le permita, tanto a nivel nacional como en escenarios internacionales $(\mathrm{ZO} / \mathrm{TO})$, realizar atención sanitaria de heridos graves in situ, y hasta que puedan ser asistidos por los equipos con capacidad de realizar soporte vital avanzado, pero sin que ello suponga la invasión del ámbito competencial propio del personal facultativo ${ }^{(4)}$.

Para tal fin, y de acuerdo con las previsiones de la Ley Orgánica 5/2005, de 17 de noviembre, de la Defensa Nacional, donde se faculta al Gobierno y al Ministro de Defensa, a dictar cuantas disposiciones sean necesarias para el desarrollo y aplicación de la misma, se promulga el Real Decreto 230/2017, de 10 de marzo, por el que se regulan las competencias y cometidos de apoyo a la atención sanitaria del personal militar no regulado por la Ley 44/2003, de 21 de noviembre, de ordenación de las profesiones sanitarias, en el ámbito estrictamente militar, aún pendiente de desarrollo pormenorizado mediante Orden Ministerial.

De este modo, se configura un modelo de apoyo sanitario que permite disponer de personal militar formado y capacitado asegurando una respuesta inmediata en situaciones excepcionales en ausencia de médicos o enfermeros, realizando las maniobras pertinentes que permiten la estabilización del trauma de combate y la atención posterior por parte de los facultativos especializados.

\section{LA FORMACIÓN EN TCCC Y LA NAEMT}

Desde el momento en que los primeros militares españoles fueron desplegados en misiones en el exterior, se suscitó la cuestión sobre si el nivel de respuesta de los sanitarios militares no facultativos estaban a la altura de los de otros países que participaban en aquellas. Es decir, se habría el debate sobre la mejor y más cualificada formación que podía dispensarse a los mismos, así como a los controles a la formación reglada en ese entorno, hasta entonces desconocido y, además, carente de una normativa nacional específica.

En este contexto, la National Association of Emergency Medical Technicians (NAEMT) es una asociación norteamericana que agrupa los intereses de los profesionales de las emergencias médicas (lo que en Europa denominaríamos como otros «primeros intervinientes») y que proporcionan atención médica prehospitalaria ya sea urgente, emergente o preventiva.

NAEMT se ha convertido, entonces, y hasta el momento, en el referente principal de capacitación profesional y estándar de acreditación para certificar y evaluar la destreza y capacidad de las unidades o elementos emergentes en las técnicas que comprende el TCCC.

\footnotetext{
(4) Una vez asistido por los NC3's, y para prestar los conocimientos suministrados por la doctrina del TCCC, e imprescindibles para salvaguardar la vida, estabilizado el herido por personal, ahora sí, facultativo del E.T., se impone su traslado a una instalación con capacidad de cirugía de control de daños o ROLE 2; o, en su caso a un ROLE 3, con más especialidades quirúrgicas, siempre que se encuentre dentro del radio de acción establecido y según la gravedad de los heridos. Una vez intervenido, podrá ser trasladado para su tratamiento definitivo, y a través de evacuación estratégica, al ROLE 4, como en España el recientemente bautizado Hospital Central de la Defensa Gómez Ulla, Centro Sanitario de Vida y Esperanza.
} 


\section{Cuestiones jurídicas controvertidas sobre las técnicas del «Tactical Combat Casualty Care»...}

De acuerdo con lo anterior, por Resolución 420/2838249/20, de 4 de septiembre, de la Secretaría General Técnica del Ministerio de defensa, se ha hecho público un Convenio, entre la empresa J\&E Diforsa y el Ministerio de Defensa, para impartir formación especializada en el ámbito sanitario TCCC, adiestrando y auditando, en su caso, a las unidades sanitarias de las FAS que deseen convertirse en Centros de Entrenamiento Autorizados NAEMT.

Se trata, en última instancia, de aumentar la capacidad de atender el trauma en combate aportado formación y mejor calidad en la asistencia del personal sanitario NC3 (no facultativo).

\section{LAS CONCRETAS COMPETENCIAS DE LOS EGRESADOS DE LOS CURSOS TCCC}

La preocupación de la necesidad de contar con militares españoles especializados en esta materia en operaciones internacionales es antigua y a ella responden las publicaciones de «Doctrina sanitaria conjunta»(2003), el OR7-603 «Orientaciones sanidad en operaciones» (2003) del Ejército de Tierra (ET), y el documento «Actualización de los Escalones de Sanidad en Operaciones y criterios para elaboración de los módulos de Plantilla Orgánica para el ciclo 2008-2010», entre otros.

Aunque el Real Decreto citado elude cualquier mención al concepto de TCCC, no cabe duda de que el resultado del programa, formativo y competencial, que describe engloba, en algunos aspectos al mismo, al menos, en su nivel más avanzado (NC3). Así, con acierto el legislador prefiere utilizar la más genérica denominación de "personal de apoyo avanzado a la atención sanitaria en operaciones» evitando las más controvertidas denominaciones de «paramédico» o "parasanitario» que no tienen cabida en la normativa española que regula las profesiones sanitarias.

Hasta ese momento, la figura que más se parecía de las contempladas en nuestro ordenamiento jurídico viene determinado por el denominado Técnico de Emergencias Sanitarias previsto en el Real Decreto 1397/2007, de 29 de octubre, quedando limitada la competencia general de los así titulados en trasladar al paciente al centro sanitario, prestar atención básica sanitaria y psicológica en el entorno pre-hospitalario, llevar a cabo actividades de tele-operación y tele -asistencia sanitaria, y colaborar en la organización y desarrollo de los planes de emergencia, de los dispositivos de riesgo previsibles y de la logística sanitaria ante una emergencia individual, colectiva o catástrofe.

El Real Decreto 23072017, describe la existencia de hasta tres niveles de formación sanitaria del combatiente de las FAS: básico (NC1), medio (NC2) y avanzado (NC3). Corresponde a este NC3, como personal específico formado, actuar en situaciones de particular aislamiento o en apoyo directo al personal facultativo en elementos de asistencia sanitaria de operaciones.

Muy consciente el legislador de la tensión que la aparición de estos nuevos intervinientes o personal de apoyo a la atención sanitaria puede generar, explicita en el Real Decreto que resulta de aplicación exclusiva dicha norma, únicamente, en las Fuerzas Armadas, durante el desarrollo de las operaciones militares y su preparación, en situaciones de aislamiento o entorno hostil, y en ausencia de personal facultativo, o en cometidos de apoyo al mismo, en el contexto operaciones militares de las que define el artículo 16 de la Ley Orgánica 5/2005, de 27 de noviembre, de la Defensa Nacional ${ }^{(5)}$.

\section{LA DELGADA LÍNEA ROJA CON EL INTRUSISMO PROFESIONAL}

Todo lo anterior conduce, inexorablemente, a un debate jurídico muy cualificado acerca de si todos esos conocimientos y habilidades adquiridos por nuestros soldados sanitarios (TC3) ya descritos, muchos de los cuales representan verdaderos actos facultativos (administrar fármacos, coger vías intravenosas o intraóseas, etc.) reservados jurídicamente a los graduados universitarios en enfermería o licenciados en medicina, podrían ser legalmente aplicados por personal que no estuviesen en posesión de la correspondiente titulación.

En este orden de cosas, el ordenamiento penal español describe el delito de intrusismo como una conducta de naturaleza falsaria, que trata de proteger a la ciudadanía de aquellas personas que, sin título habilitante, ni cualificación suficiente, ejercen una actividad profesional para la que no están habili$\operatorname{tados}^{(6)}$. La presunción de aptitud e idoneidad lo da la titulación, que se erige como requisito administrativo, sin el cual, si se ejerce una actividad profesional sin el título resulta un ilícito penal con independencia del resultado, o de la aptitud y actitud para la actividad, ya que ello entra en otro terreno distinto, al no tratarse de una buena o mala praxis profesional, sino del ejercicio profesional «sin habilitación» como presupuesto administrativo $^{(7)}$.

El intrusismo profesional como práctica delictual es perseguible desde el art. 403 del Código Penal y aparece descrito en los siguientes términos: "quien ejerza actos propios de una profesión sin poseer el correspondiente título académico expedido

\footnotetext{
(5) Según el citado artículo, el cumplimiento de las misiones de las FAS y el desarrollo de su contribución complementaria o subsidiaria de interés público requieren realizar diferentes tipos de operaciones, tanto en territorio nacional como en el exterior, que pueden conducir a acciones de prevención de conflictos o disuasión, de mantenimiento de la paz, actuaciones en situaciones de crisis y, en su caso, de respuesta a la agresión. En particular, las operaciones pueden consistir en: a) La vigilancia de los espacios marítimos; b) La colaboración en operaciones de mantenimiento de la paz y estabilización internacional c) El apoyo a las Fuerzas y Cuerpos de Seguridad del Estado en la lucha contra el terrorismo y a las instituciones y organismos responsables de los servicios de rescate terrestre, marítimo y aéreo, en las tareas de búsqueda y salvamento; d) La respuesta militar contra agresiones que se realicen utilizando aeronaves con fines terroristas; e) La colaboración con las diferentes Administraciones públicas en los supuestos de grave riesgo, catástrofe, calamidad u otras necesidades públicas, conforme a lo establecido en la legislación vigente; f) La participación con otros organismos nacionales e internacionales para preservar la seguridad y el bienestar de los ciudadanos españoles en el extranjero.

(6) El bien jurídico protegido por el tipo penal -dice la Sentencia del Tribunal Supremo de 14 de octubre de 2011, está caracterizado por su carácter pluriofensivo por a saber: a) el privado de quien recibe la prestación profesional del intruso; b) la corporación profesional a la que afecta la conducta intrusa; y, c) la sociedad en su interés público en que sean idóneas las personas que ejercen determinadas profesiones para las que el Estado reglamenta el acceso a la actividad.

(7) Vid., la STS 167/2020, 19 de mayo de 2020.
} 
o reconocido en España incurrirá en la pena de multa de doce a veinticuatro meses» ${ }^{(8)}$.

El tipo penal que describe el delito de intrusismo presenta una estructura de ley penal en blanco («títulos oficiales», «actos propios de una profesión», etc), esto es, de normas penales incompletas en las que la conducta o la consecuencia jurídico-penal no se encuentre agotadoramente descrita en ellas, debiendo acudirse, para su integración, a otra norma distinta ${ }^{(9)}$. No obstante, y a los efectos que aquí nos interesan, debe entenderse por actos propios de una profesión aquellos que específicamente están reservados a una profesión sanitaria, quedando excluidas de su realización aquellas personas que carezcan de la titulación precisa $^{(10)}$

La tensión de los habilitados TC3 con los titulares de las profesiones sanitarias contemplados en la Ley 44/2003, de 21 de enero de 2003, se colige con claridad meridiana, y sin necesidad de ahondar pormenorizadamente en las concretas competencias profesionales de cada sector, y que escapan al propósito de este estudio.

\section{LA PREVISIÓN NORMATIVA COMO CAUSA DE EXENCIÓN DE LA RESPONSABILIDAD Y EL ESTADO DE NECESIDAD}

Como acabamos de poner de relieve, ninguna duda cabe que las prácticas que se describen en el Real Decreto 230/2017, de 10 de marzo, constituyen técnicas expresamente reservadas a enfermeros $^{(11)}$ y técnicos de emergencia ${ }^{(12)}$, pero también, incluso

(8) En el Boletín Oficial del Estado, de 31 de Marzo de 2015, se publicó la Ley Orgánica 1/2015, de 30 de marzo, por la que se modifica la Ley Orgánica 10/1995, de 23 de noviembre del Código Penal, cuya entrada en vigor tuvo lugar el pasado 1 de julio tal y como establecía su disposición final octava con lo que se dobla lo que establecía hasta ahora la L. O. 10/1995, de 23 de noviembre. Además, supone una mayor concreción en la redacción actual del tipo agravado, incluyendo como particularidad cuando esta actividad «se realiza en un local o establecimiento abierto al público en el que se anuncia la prestación de servicios propios de la profesión» estableciendo la pena de prisión de entre seis meses y dos años. Con ello se pretende hacer frente a situaciones que no estaban expresamente previstas en la legislación pasada, pero que son frecuentes en la práctica y suponen un evidente fraude que debe sancionarse.

(9) Los elementos que configuran el delito de intrusismo son dos: a) La realización o ejecución de actos propios de una profesión para la que sea preciso título oficial, o reconocido por disposición legal o Convenio Internacional (título académico o título oficial de capacitación); y, b) Violación antijurídica de la normativa extrapenal ordenadora de la profesión invadida y, en particular, de aquel sector que reglamenta la concesión y expedición de la titularidad que faculta para el ejercicio de la actividad profesional que se enjuicia.

(10) «Acto propio de una profesión» es aquél que específicamente está atribuido a unos profesionales concretos con terminante exclusión de las demás personas y habrán de ser, precisamente, normas jurídicas las que determinen qué deban ser actos propios de una profesión para cuyo ejercicio habilite un título oficial

(11) Según el artículo 7.2 a) de la citada Ley de Profesiones Sanitarias, corresponde a los Diplomados universitarios en Enfermería la dirección, evaluación y prestación de los cuidados de Enfermería orientados a la promoción, mantenimiento y recuperación de la salud, así como a la prevención de enfermedades y discapacidades.

(12) No hay que olvidar, que entre sus competencias se encuentra la UC0360 2 consistente en colaborar en la organización y el desarrollo de la logística sanitaria en escenarios con múltiples víctimas y catástrofes, asegurando el a médicos ${ }^{(13)}$ que en principio será realizado por personal militar con formación en TCCC o NC3 (señala la norma).

Ahora bien, el contexto competencial para la práctica de dichas técnicas se circunscribe espacial y temporalmente a escenarios operativos (en principio pensado para desarrollarse fuera del territorio nacional) como ámbito propio ( $\mathrm{ZO} / \mathrm{TO})$. Ahora bien, con carácter previsor el legislador habla de modo genérico de «escenarios operativos» en los que la citada evidencia científica avala el impacto que tiene sobre la supervivencia del herido en combate la doctrina del TCCC.

No sería desorbitado pensar, entonces, que tal doctrina pudiera ser aplicada en territorio nacional o en operaciones que se lleven a cabo, incluso, en suelo español, como recientemente se ha puesto de manifiesto a propósito de la OP. BALMIS. Ahora bien, siempre ceñidos a los presupuestos que describe el Real Decreto 230/2017 (arts. 2 y 4), esto es, en situaciones de aislamiento o entorno hostil y ausencia de personal facultativo para atender las lesiones que producen un mayor número de muertes como son las hemorragias masivas, el neumotórax a tensión y la obstrucción de la vía aérea en un contexto de múltiples víctimas.

En relación con lo anterior, la legislación penal española, inmediatamente después de configurar la legítima defensa, incluye entre los supuestos que sirven de base a la aplicación de una circunstancia eximente de responsabilidad penal aquellas situaciones de necesidad en las que el sujeto ha de infringir un deber o lesionar un bien jurídico con el objeto de salvaguardar otro ${ }^{(14)}$.

Para la aplicación de la eximente del art. 20.5 a la que nos referimos, y que podría amparar la actuación de nuestros sanitarios militares, el Código penal se limita a exigir que se actúe en estado de necesidad; pero no define qué se ha de entender por el mismo. De acuerdo con la doctrina jurídica y jurisprudencial la misma pudiera perfilarse a partir de los postulados siguientes:

1. En primer lugar debe concurrir un peligro actual para un bien jurídico. Esto es, la vida o la integridad física del militar herido (o del civil asistido por las FAS), llevando a cabo un juicio de previsibilidad ex ante, y que ha de aparecer como no absolutamente improbable la producción de la lesión del bien jurídico.

2. El origen del peligro puede provenir de la acción de fuerzas enemigas pero también de los derivados de riesgos antrópicos, tecnológicos o naturales.

3. Los bienes jurídicos en peligro pueden ser propios (caso de practicarse las técnicas en el propio interviniente), o ajenos (ámbito natural del NC3).

abastecimiento y la gestión de recursos y apoyando las labores de coordinación en situaciones de crisis.

(13) De acuerdo con el art. 6.2 a) Ley de Profesiones Sanitarias establece que corresponde a los Licenciados en Medicina la indicación y realización de las actividades dirigidas a la promoción y mantenimiento de la salud, a la prevención de las enfermedades y al diagnóstico, tratamiento, terapéutica y rehabilitación de los pacientes, así como al enjuiciamiento y pronóstico de los procesos objeto de atención.

(14) El art. 20.5 CP recoge la eximente de estado de necesidad en los siguientes términos: «Están exentos de responsabilidad criminal: [...] 5. El que, en estado de necesidad, para evitar un mal propio o ajeno lesione un bien jurídico de otra persona o infrinja un deber, siempre que concurran los siguientes requisitos: Primero. Que el mal causado no sea mayor que el que se trate de evitar. Segundo. Que la situación de necesidad no haya sido provocada intencionadamente por el sujeto. Tercero. Que el necesitado no tenga, por su oficio o cargo, obligación de sacrificarse.» 


\section{Cuestiones jurídicas controvertidas sobre las técnicas del «Tactical Combat Casualty Care»...}

4. El mal ha de aparecer como inminente o, al menos, el conflicto debe resultar irresoluble con el transcurso del tiempo, esto es, de no actuarse se agravarán las lesiones de manera irresoluble y hacia una muerte segura.

5. Se ha de tratar de un mal grave, como es el peligro de la vida o, al menos, una situación de riesgo vital.

6. Para evitar el mal ha de ser preciso lesionar otro bien jurídico o infringir un deber, como es la lesión menor de la integridad física (v. gr., intervención cricotiroidea) o el intrusismo en las técnicas de las profesiones sanitarias.

Atendiendo a los anteriores requisitos y, con independencia del amparo legal en los supuestos que prevé el Real Decreto 230/2017, la conducta realizada por los NC3 pudiera quedar amparada, convenientemente valorada por los tribunales y para el caso concreto, en los supuestos de exigencia de responsabilidad penal, como un caso de situación de necesidad amparado por el derecho que le eximiría de la misma ${ }^{(15)}$.

En la esencia de la situación de necesidad, el mal no ha de ser evitable por un procedimiento menos perjudicial, ilícito o lícito.

\section{CONCLUSIONES}

1. El TCCC ha revelado que la adecuada atención por parte del personal militar debidamente adiestrado aumenta de modo considerable las posibilidades de supervivencia del herido en combate como ha puesto de relieve la evidencia científica.

2. Para que alcance su máxima eficacia se debe contar con un personal militar altamente cualificado, así como de formadores previamente adiestrados y acreditados por la NAEMT. Para ello, no se excluye la posibilidad de convenir instrumentos jurídicos de colaboración entre el Ministerio de Defensa y aquellas organizaciones privadas que gocen del estándar de calidad requerido y convenientemente certificado.

3. El panorama de las profesiones sanitarias regladas en España desde la perspectiva penal, dada algunas de las técnicas que se aplican en el TCCC, pueden suponer un riesgo de comisión presunta de un delito de intrusismo, la cual debiera quedar superada, en el hipotético escenario judicial, a tenor de la novedosa regulación legal expuesta y la doctrina del estado de necesidad exculpante descrito en las páginas que preceden.

4. La normativa que regula las competencias sobre las profesiones sanitarias debiera ser interpretada, a salvo la opinión de los órganos de control judicial sobre esta materia, en el sentido más favorable para poder capacitar a ese personal sanitario militar (no facultativo), y ajeno a su regulación, de manera que permita dotarles de las habilidades adecuadas y que redundan, en última instancia, en las mejor asistencia al herido en combate y la salvaguarda de su vida en ausencia de facultativos adecuados en ambiente hostil.

(15) En cualquier caso, los daños a bienes de terceros deberán ser abonados siguiendo las previsiones del art. 118.1.3 CP. Según el mismo: «En el caso del número $5^{\circ}$ serán responsables civiles directos las personas en cuyo favor se haya precavido el mal, en proporción al perjuicio que se les haya evitado, si fuera estimable o, en otro caso, en la que el Juez o Tribunal establezca según su prudente arbitrio. 